\title{
Cross-sectional association of dietary patterns with insulin-resistant phenotypes among adults without diabetes in the Framingham Offspring Study
}

\author{
Enju Liu ${ }^{1,2}$, Nicola M. McKeown ${ }^{1,2 *}$, P. K. Newby ${ }^{3}$, James B. Meigs ${ }^{4}$, Ramachandran S. Vasan ${ }^{5}$, \\ Paula A. Quatromoni ${ }^{6}$, Ralph B. D’Agostino ${ }^{7}$ and Paul F. Jacques ${ }^{1,2}$ \\ ${ }^{1}$ Jean Mayer US Department of Agriculture Human Nutrition Research Center on Aging, Tufts University, Boston, MA, USA \\ ${ }^{2}$ Gerald J. and Dorothy R.. Friedman School of Nutrition Science and Policy, Tufts University, Boston, MA, USA \\ ${ }^{3}$ Department of Pediatrics, Boston University School of Medicine and Department of Epidemiology, Boston University School \\ of Public Health, Boston, MA, USA \\ ${ }^{4}$ The General Medicine Division and Department of Medicine, Massachusetts General Hospital, Boston, MA, USA \\ ${ }^{5}$ Framingham Heart Study, Boston University School of Medicine, Boston, MA, USA \\ ${ }^{6}$ Department of Health Sciences, Sargent College of Health and Rehabilitation Sciences, Boston University, Boston, MA, USA \\ ${ }^{7}$ Department of Biostatistics, Boston University School of Public Health, Boston, MA, USA
}

(Received 21 July 2008 - Revised 23 October 2008 - Accepted 10 December 2008 - First published online 16 February 2009)

\begin{abstract}
Cluster analysis is a valuable tool for exploring the health consequences of consuming different dietary patterns. We used this approach to examine the cross-sectional relationship between dietary patterns and insulin-resistant phenotypes, including waist circumference, BMI, fasting insulin, $2 \mathrm{~h}$ post-challenge insulin, insulin sensitivity index $\left(\mathrm{ISI}_{0,120}\right)$, HDL-cholesterol, TAG and blood pressure, using data from the fifth examination cycle of the Framingham Offspring Study. Among 2875 participants without diabetes, we identified four dietary patterns based on the predominant sources of energy: 'Fruits, Reduced Fat Dairy and Whole Grains', 'Refined Grains and Sweets', 'Beer' and 'Soda'. After adjusting for multiple comparisons and potential confounders, compared with the 'Fruits, Reduced Fat Dairy and Whole Grains' pattern, the 'Refined Grains and Sweets' pattern had significantly higher mean waist circumference $(92.4 v .90 .5 \mathrm{~cm} ; P=0.008)$ and BMI $\left(27.3 v .26 .6 \mathrm{~kg} / \mathrm{m}^{2} ; P=0.02\right)$; the 'Soda' pattern had significantly higher mean fasting insulin concentration $(31.3$ v. $28.0 \mu \mathrm{U} / \mathrm{ml} ; P \leq 0.001)$; the 'Beer' pattern had significantly higher mean HDL-cholesterol concentration $(1.46$ v. $1.31 \mathrm{mmol} / 1 ; P<0.001)$. No associations were observed between dietary patterns and $\mathrm{ISI}_{0,120}$, TAG, and systolic or diastolic blood pressure. Our findings suggest that consumption of a diet rich in fruits, vegetables, whole grains and reducedfat dairy protects against insulin-resistant phenotypes and displacing these healthy choices with refined grains, high-fat dairy, sweet baked foods, candy and sugar-sweetened soda may promote insulin-resistant phenotypes.
\end{abstract}

Dietary patterns: Cluster analysis: Insulin-resistant phenotypes: Framingham Offspring Study

Insulin resistance is a common pathological state in which target cells (liver, muscle and adipose tissue) fail to respond to normal levels of circulating insulin ${ }^{(1)}$. Concomitant conditions that are associated with insulin resistance include impaired glucose tolerance, impaired fasting glucose, elevated insulin concentrations, low HDL-cholesterol concentrations and elevated TAG concentrations ${ }^{(2)}$. Diet is believed to play a key role in the development of insulin resistance. To date, various aspects of diet, such as fat, carbohydrate, fibre, whole grains, $\mathrm{Mg}$, glycaemic index and load ${ }^{(3-7)}$, have been related to insulin resistance. Most observational studies relate individual nutrients or foods to various outcomes and although these studies contribute to our understanding on the role of diet in disease, there are several potential limitations.
First, due to the high correlation between components of a diet, it is often difficult to examine the separate effect for a specific nutrient or food. Second, as interactions may exist between components of a diet, the findings of single-nutrient or food analysis may be misleading. Finally, the effect of a single nutrient or food on a disease outcome or risk factor may be too small to detect, particularly in smaller cohorts ${ }^{(8,9)}$.

The use of dietary patterns to capture the overall dietary habits of populations has received much attention in recent years. Instead of focusing on the effect of individual nutrients or foods, a dietary pattern approach examines the effect of an overall diet on health outcomes, thereby representing a complex combination of foods and nutrients ${ }^{(10)}$. Thus, from a public health perspective, results from dietary pattern analysis

Abbreviations: ISI $_{0,120}$, insulin sensitivity index; OGTT, oral glucose tolerance test.

* Corresponding author: Dr Nicola M. McKeown, fax +1 617556 3344, email nicola.mckeown@tufts.edu 
can be easily translated and incorporated into dietary guidelines for the public ${ }^{(9)}$. A number of observational studies have examined the association between dietary patterns and either the metabolic syndrome, or components of the metabolic syndrome ${ }^{(11-16)}$. Although insulin resistance is a key underlying risk factor associated with this syndrome, few studies have used a dietary pattern approach to directly examine the relationship between diet and surrogate measures of insulin resistance in healthy adults ${ }^{(11,17)}$. To our knowledge, no studies have evaluated dietary patterns derived by cluster analysis in relation to insulin resistance in a population without diabetic patients. The purpose of the present study was to determine if specific dietary patterns derived by cluster analysis are related to insulin-resistant phenotypes among adults without diabetes.

\section{Experimental methods}

\section{Participants}

The present investigation examined the cross-sectional relationship between dietary patterns and insulin-resistant phenotypes using data from the Framingham Offspring Study. The Framingham Study was initiated in 1948 as a longitudinal population-based study of CVD. In 1971, 5135 offspring of original participants of the Framingham Study and spouses of the offspring were recruited to participate in the Framingham Offspring Study. Members of the Framingham Offspring Study have returned on average every 4 years for a physical examination, questionnaires, laboratory tests, and assessment of cardiovascular and other risk factors ${ }^{(18)}$. During the fifth examination cycle (between 1991 and 1995), a total of 3799 participants underwent a standardised medical history and physical examination. Valid FFQ were available for 3418 participants. Participants were excluded from this analysis if they had previously diagnosed ( $n$ 212) diabetes, or if their fasting glucose concentrations were $\geq 7.0 \mathrm{mmol} / \mathrm{l}$, or $2 \mathrm{~h}$ post-challenge glucose concentrations were $\geq 11.1 \mathrm{mmol} / \mathrm{l}$ ( $n$ 134). We also excluded those who were taking cholesterollowing medications ( $n$ 197). After exclusions, the final sample size was 2875 participants (1278 men and 1597 women). The institutional review boards for human research at Boston University and Tufts Medical Center reviewed and approved all study protocols and procedures.

\section{Dietary assessment}

Usual dietary intake for the previous year was assessed at the fifth examination using a 126-item semi-quantitative $\mathrm{FFQ}^{(19)}$. The questionnaires were mailed to participants before the examination, and the participants were asked to bring the completed questionnaire with them to their examination at the Framingham Heart Study. The FFQ consists of a list of foods with a standardised serving size and a selection of nine frequency categories ranging from never or less than one serving per month to more than six servings/d. Separate questions about the use of vitamin and mineral supplements and the type of breakfast cereal most commonly consumed were also included in the FFQ. FFQ with reported energy intakes less than $2.51 \mathrm{MJ} / \mathrm{d}(600 \mathrm{kcal} / \mathrm{d})$ for men and women, or more than $16.74 \mathrm{MJ} / \mathrm{d}(4000 \mathrm{kcal} / \mathrm{d})$ for women or more than $17.57 \mathrm{MJ} / \mathrm{d}(4200 \mathrm{kcal} / \mathrm{d})$ for men, or with more than twelve food items left blank were considered invalid. Nutrient intakes were calculated by multiplying the frequency of consumption of each unit of food from the FFQ by the nutrient content of the specified portion. The relative validity of the FFQ has been reported for both nutrients and foods ${ }^{(19,20)}$. Individual foods from the 126-item FFQ were collapsed into forty food groups based on the similarity of food and nutrient composition (Table 1). Dietary data were used to calculate the average glycaemic index of a subject's diet, which is the mean glycaemic index of all the food items in the FFQ weighted by the content of carbohydrate from each food item. The dietary guidelines adherence index, a measure of adherence to the key dietary intake recommendations in the 2005 Dietary Guidelines for Americans, was calculated as a measure of diet quality. The score ranged from $2 \cdot 5$ to 17.5 with a mean of $9 \cdot 1$ in the present study population $^{(21)}$.

\section{Outcome measurements}

Height, weight and waist circumference were measured in the standing position by a trained technician. BMI was calculated as weight in kilograms divided by the square of height in meters. Blood samples were drawn after the participants had fasted for at least $8 \mathrm{~h}$ for the measurement of fasting glucose and insulin, and lipids. A $75 \mathrm{~g}$ oral glucose tolerance test (OGTT) was administered according to WHO standards to measure the $2 \mathrm{~h}$ post-challenge glucose and insulin ${ }^{(22)}$. Plasma glucose concentrations were measured in fresh specimens with a hexokinase reagent kit (A-gent glucose test; Abbott Laboratories, Inc., South Pasadena, CA, USA); the intra-assay $\mathrm{CV}$ was $<3 \%$. Plasma insulin concentrations were measured using the Coat-A-Count ${ }^{125}$ I-radioimmunoassy (Diagnostic Products, Los Angeles, CA, USA); this assay has cross-reactivity with proinsulin at the midcurve of $40 \%$, the intra- and inter-assay CV were 5-10\%. Insulin sensitivity index $\left(\right.$ ISI $_{0,120}$ ) was calculated using the following formula ${ }^{(23)}$ :

$$
\mathrm{ISI}_{0,120}=(\mathrm{m} / \mathrm{MPG}) / \log \mathrm{MSI}
$$

where

$$
\mathrm{m}=(75000 \mathrm{mg}+(\text { fasting glucose }-2 \mathrm{~h} \text { post }
$$

- OGTT glucose) $\times 0 \cdot 19 \times$ body weight $(\mathrm{kg})) / 120 \mathrm{~min}$,

MPG is the mean of fasting and $2 \mathrm{~h}$ post-OGTT glucose concentrations $(\mathrm{mg} / \mathrm{dl})$ and MSI is the mean of fasting and $2 \mathrm{~h}$ post-OGTT insulin concentrations (mU/l).

Serum lipid measures included the enzymic measurement of $\mathrm{TAG}^{(24)}$ and the measurement of the HDL-cholesterol fraction after precipitation of LDL- and VLDL-cholesterol with dextran sulfan magnesium ${ }^{(25)}$. Blood pressure was measured twice after the participants sat for $5 \mathrm{~min}$ or more.

\section{Covariates}

Physical activity was measured using a standardised questionnaire to determine estimates of activity based on a $24 \mathrm{~h}$ history. A physical activity score was calculated from the number of hours spent doing specific activities that were 
Table 1. Percentage energy contribution from food groups across the four dietary patterns among 2875 non-diabetic adults participating in the Framingham Offspring Study at the fifth examination cycle*

(Mean values with their standard errors)

\begin{tabular}{|c|c|c|c|c|c|c|c|c|}
\hline & \multicolumn{2}{|c|}{$\begin{array}{l}\text { Fruits, Reduced Fat } \\
\text { Dairy and Whole } \\
\text { Grains }(n 577)\end{array}$} & \multicolumn{2}{|c|}{$\begin{array}{l}\text { Refined Grains } \\
\text { and Sweets } \\
(n 1846)\end{array}$} & \multicolumn{2}{|c|}{$\begin{array}{l}\text { Beer } \\
(n \text { 242) }\end{array}$} & \multicolumn{2}{|c|}{$\begin{array}{l}\text { Soda } \\
(n 210)\end{array}$} \\
\hline & Mean & SE & Mean & SE & Mean & SE & Mean & SE \\
\hline High-fat dairy & $5 \cdot 1^{a}+$ & 0.2 & $6 \cdot 8^{\mathrm{b}} \ddagger$ & 0.1 & $6 \cdot 4^{\mathrm{b}}$ & 0.3 & $6 \cdot 1^{\mathrm{a}, \mathrm{b}}$ & 0.4 \\
\hline Reduced-fat dairy & $5 \cdot 1^{a} \ddagger$ & 0.2 & $3.8^{\mathrm{b}}$ & 0.1 & $2 \cdot 8^{\mathrm{c}}$ & 0.3 & $2 \cdot 2^{\mathrm{c}} \dagger$ & 0.3 \\
\hline High-fat dairy desserts & $0.8^{\mathrm{a}}+$ & 0.1 & $1 \cdot 2^{b} \ddagger$ & 0.04 & $0.9^{\mathrm{a}, \mathrm{b}}$ & 0.1 & $1 \cdot 2^{b} \ddagger$ & 0.1 \\
\hline Reduced-fat dairy desserts & 0.4 & 0.1 & 0.4 & 0.03 & 0.3 & 0.1 & 0.3 & 0.1 \\
\hline Margarine & $1 \cdot 2^{\mathrm{a}, \mathrm{b}}$ & 0.1 & $1.4^{\mathrm{a}}$ & 0.04 & $1 \cdot 1^{b} t$ & 0.1 & $1.5^{\mathrm{a}, \mathrm{b}} \neq$ & 0.1 \\
\hline Miscellaneous fats & 0.1 & 0.02 & 0.1 & 0.01 & 0.1 & 0.03 & 0.1 & 0.04 \\
\hline Fruit juices & $5 \cdot 5^{\mathrm{a}} \neq$ & 0.1 & $2 \cdot 2^{b}$ & 0.1 & $1.9^{\mathrm{b}} \dagger$ & 0.2 & $2 \cdot 0^{\mathrm{b}}$ & 0.2 \\
\hline Fruits & $8 \cdot 5^{\mathrm{a}} \neq$ & 0.2 & $5 \cdot 0^{\mathrm{b}}$ & 0.1 & $3 \cdot 5^{\mathrm{c}}$ & $0 . \overline{3}$ & $3 \cdot 2^{c} \dagger$ & $0 . \overline{3}$ \\
\hline Fruit drinks & $2 \cdot 8^{a} \ddagger$ & 0.1 & $1 \cdot 6^{\mathrm{b}, \mathrm{c}}$ & 0.1 & $1 \cdot 2^{\mathrm{b}}+$ & 0.2 & $2 \cdot 1^{\mathrm{a}, \mathrm{c}}$ & 0.2 \\
\hline Tofu and beans & $0.9^{a} \neq$ & 0.04 & $0.6^{\mathrm{b}}$ & 0.02 & $0.6^{\mathrm{b}}$ & 0.1 & $0.5^{\mathrm{b}} \dagger$ & 0.1 \\
\hline Nuts and seeds & 1.8 & 0.1 & 1.9 & 0.1 & $1 \cdot 8$ & 0.2 & 1.6 & 0.2 \\
\hline Vegetables & $5 \cdot 0^{\mathrm{a}} \ddagger$ & 0.1 & $3.9^{b}$ & 0.01 & $3 \cdot 6^{\mathrm{b}, \mathrm{c}}$ & 0.1 & $3 \cdot 2^{c} \dagger$ & 0.1 \\
\hline Starchy vegetables & 4.9 & 0.1 & 4.9 & 0.1 & 4.8 & 0.2 & 4.6 & 0.2 \\
\hline Eggs & $0 \cdot 8$ & 0.04 & 0.8 & 0.02 & 0.7 & 0.1 & 0.8 & 0.1 \\
\hline Poultry & $6 \cdot 0^{a} \ddagger$ & 0.2 & $5 \cdot 5^{\mathrm{b}}$ & 0.1 & $4 \cdot 6^{\mathrm{c}}$ & 0.3 & $4 \cdot 4^{\mathrm{C}} \dagger$ & 0.3 \\
\hline Processed meat & $1 \cdot 2^{a}+$ & 0.1 & $1.9^{b}$ & 0.01 & $2 \cdot 0^{\mathrm{b}} \ddagger$ & 0.1 & $2 \cdot 0^{\mathrm{b}} \ddagger$ & $0 \cdot 1$ \\
\hline Liver & $0.05^{\mathrm{a}} \dagger$ & 0.01 & $0.09^{\mathrm{b}} \ddagger$ & 0.01 & $0.07^{\mathrm{a}, \mathrm{b}}$ & 0.02 & $0.07^{\mathrm{a}, \mathrm{b}}$ & 0.02 \\
\hline Meat & $4 \cdot 8^{a}+$ & 0.2 & $6 \cdot 6^{\mathrm{b}}$ & 0.1 & $6 \cdot 4^{\mathrm{b}}$ & 0.3 & $6 \cdot 9^{b} \ddagger$ & 0.3 \\
\hline Fish and other seafood & $3 \cdot 3^{a} \neq$ & 0.1 & $2 \cdot 4^{\mathrm{b}}$ & 0.01 & $2 \cdot 1^{\mathrm{b}, \mathrm{c}}$ & $0 \cdot 1$ & $2 \cdot 0^{c} \dagger$ & 0.1 \\
\hline Whole-grain cereal & $2 \cdot 2^{a} \neq$ & 0.1 & $1 \cdot 6^{\mathrm{b}}$ & 0.1 & $0.9^{\mathrm{c}}$ & 0.2 & $0.8^{\mathrm{C}} \dagger$ & 0.2 \\
\hline Refined-grain cereal & $1.3^{\mathrm{a}} \neq$ & 0.1 & $0.8^{\mathrm{b}}$ & 0.01 & $0.5^{\mathrm{b}} \dagger$ & 0.1 & $0.8^{\mathrm{b}}$ & 0.1 \\
\hline Whole grains & $6 \cdot 6^{a} \ddagger$ & 0.2 & $3.7^{\mathrm{b}}$ & 0.1 & $2 \cdot 8^{\mathrm{c}}$ & 0.3 & $2 \cdot 0^{\mathrm{C}} \dagger$ & 0.3 \\
\hline Refined grains & $7 \cdot 3^{\mathrm{a}}$ & 0.2 & $9 \cdot 5^{b} \ddagger$ & 0.1 & $7 \cdot 0^{\mathrm{a}} \dagger$ & 0.3 & $7 \cdot 8^{\mathrm{a}}$ & 0.3 \\
\hline Pasta & $3 \cdot 2^{a} \neq$ & 0.1 & $3.0^{\mathrm{a}}$ & 0.1 & $2 \cdot 8^{\mathrm{a}, \mathrm{b}}$ & 0.2 & $2 \cdot 4^{\mathrm{b}} \dagger$ & 0.2 \\
\hline Chocolate & $1.0^{\mathrm{a}} \mathrm{t}$ & 0.1 & $2 \cdot 4^{\mathrm{b}}$ & 0.1 & $2 \cdot 0^{\mathrm{b}}$ & 0.2 & $2 \cdot 7^{b} \ddagger$ & 0.2 \\
\hline Candy without chocolate & $0.5^{\mathrm{a}}+$ & 0.1 & $0.9^{b} \ddagger$ & $0 \cdot 1$ & $0.5^{\mathrm{a}, \mathrm{b}} \dagger$ & 0.2 & $0.8^{\mathrm{a}, \mathrm{b}}$ & 0.2 \\
\hline Sweet baked foods & $2.9^{\mathrm{a}}+$ & 0.2 & $9 \cdot 0^{\mathrm{b}} \ddagger$ & 0.1 & $3 \cdot 9^{\mathrm{a}}$ & 0.3 & $6 \cdot 1^{\mathrm{c}}$ & 0.3 \\
\hline Miscellaneous sweets & $1.4^{\mathrm{a}}$ & 0.1 & $1.5^{\mathrm{a}}$ & 0.01 & $1 \cdot 2^{\mathrm{a}} \dagger$ & 0.1 & $2 \cdot 0^{\mathrm{b}} \ddagger$ & 0.1 \\
\hline Vegetable oils & $2 \cdot 7^{a} \ddagger$ & 0.1 & $2 \cdot 6^{\mathrm{a}}$ & 0.1 & $2 \cdot 6^{a}$ & 0.2 & $2 \cdot 0^{\mathrm{b}}+$ & 0.2 \\
\hline Chowder/cream soup & $0.4^{\mathrm{a}^{\mathrm{T}}}$ & 0.03 & $0.5^{\mathrm{b}}$ & 0.02 & $0.4^{\mathrm{a}, \mathrm{b}}$ & 0.04 & $0.4^{a, b}$ & 0.04 \\
\hline Soda & $1 \cdot 1^{a}+$ & 0.1 & $1.5^{\mathrm{b}}$ & 0.1 & $1 \cdot 7^{\mathrm{b}}$ & 0.2 & $15 \cdot 9^{c} \ddagger$ & 0.2 \\
\hline Beer & $0.9^{a}$ & 0.1 & $0.9^{a}$ & 0.1 & $18 \cdot 1^{b} \ddagger$ & 0.2 & $1 \cdot 1^{\mathrm{a}^{\mathrm{T}}}$ & $0 . \overline{2}$ \\
\hline Red wine & $0.8^{\mathrm{a}} \neq$ & 0.1 & $0.5^{\mathrm{b}}$ & 0.04 & $0 \cdot 8^{\mathrm{a}, \mathrm{b}} \ddagger$ & 0.1 & $0.3^{\mathrm{b}} \dagger$ & 0.1 \\
\hline White wine & $1 \cdot 1^{\mathrm{a}} \neq$ & 0.1 & $0.8^{\mathrm{b}}$ & 0.1 & $0 \cdot 8^{\mathrm{a}, \mathrm{b}}$ & 0.1 & $0.3^{\mathrm{c}} \dagger$ & $0 \cdot 1$ \\
\hline Liquor & $2 \cdot 1^{a} \neq$ & 0.1 & $1 \cdot 2^{\mathrm{b}} \dagger$ & 0.1 & $1.5^{\mathrm{a}, \mathrm{b}}$ & 0.2 & $1 \cdot 2^{\mathrm{b}} \dagger$ & 0.2 \\
\hline Coffee & 0.6 & 0.02 & 0.7 & 0.01 & 0.6 & 0.04 & 0.6 & 0.04 \\
\hline Mixed dishes & $3.9^{\mathrm{a}} \mathrm{t}$ & 0.2 & $5 \cdot 6^{\mathrm{b}} \neq$ & 0.1 & $4 \cdot 8^{c}$ & 0.2 & $5 \cdot 6^{\mathrm{b}, \mathrm{c}} \ddagger$ & 0.3 \\
\hline Salty snacks & $1.0^{a}+$ & 0.1 & $1.3^{\mathrm{b}^{\mathrm{T}}}$ & 0.04 & $1 \cdot 2^{a, b}$ & 0.1 & $1.5^{c} \neq$ & 0.1 \\
\hline Fried food & $0.5^{a}+$ & 0.02 & $0.6^{\mathrm{b}}$ & 0.01 & $0.6^{\mathrm{b}, \mathrm{c}}$ & 0.03 & $0.7^{C} \ddagger$ & 0.03 \\
\hline Diet soda (servings/d) & $0.6^{a} \neq$ & 0.04 & $0.6^{\mathrm{a}} \neq$ & 0.02 & $0.5^{\mathrm{a}}$ & 0.1 & $0.2^{b} \dagger$ & 0.1 \\
\hline
\end{tabular}

a,b,c,d Values within a row with unlike superscript letters are significantly different $(P<0 \cdot 05$; Tukey-Kramer's adjustment for multiple comparisons).

${ }^{*}$ Adjusted for age and sex.

tLowest mean in the row.

$\ddagger$ Highest mean in the row.

categorised (sleep, sedentary, slight activity, moderate activity and heavy activity) and weighted according to oxygen consumption required to perform them (metabolic equivalent task (MET)-h per d) ${ }^{(26)}$. Additional covariate information included age, sex, smoking dose $(0,1-15,16-25$, or $>25$ cigarettes/d) and years of education.

\section{Statistical analysis}

All statistical analyses were performed using the Statistical Analysis Systems statistical software package (version 9.1; SAS Institute, Cary, NC, USA). For cluster analysis, the PROC FASTCLUS procedure was used to derive dietary patterns. We used the K-means method to classify participants into a predetermined number of mutually exclusive, nonoverlapping groups (clusters) by comparing Euclidean distances between each subject and each cluster centre. It aims to minimise the differences within clusters while maximising the differences between clusters ${ }^{(27,28)}$. In order to minimise the effect of outliers on the cluster analysis, the FASTCLUS procedure was performed on a predefined number of clusters (n 20). Clusters with less than five individuals were removed and the remaining clusters were used as seeds for the subsequent analysis. The analysis was re-run with varying number of clusters, ranging from three to six using the seeds. In addition the analysis was re-run using sex-specific 
clusters. However, due to the observation of similar clusters in men and women and lack of any significant sex interaction with respect to our current clusters and outcomes, we chose to retain the clusters that included both men and women to maximise statistical power.

The four-cluster set (Table 1) was selected as the final because it provided the most distinct eating patterns. The food groups, represented by the percentage of energy contributed from each food group, were the input variables in the cluster analysis. We decided to treat the food group variables as a percentage of total energy as this helps to remove extraneous variation due to differences in sex, age, body size and physical activity ${ }^{(29)}$. The 'low-calorie soda' (i.e. diet soda) food group was excluded from cluster analysis because it does not provide energy, leaving thirty-nine food groups for inclusion in the cluster analysis. For descriptive purposes only, daily diet soda consumption was presented as servings per $\mathrm{d}$.

To describe food intake across the four clusters (dietary patterns), the age- and sex-adjusted means of energy contribution from each food group were calculated using analysis of covariance (PROC GLM; SAS Institute, Inc.). Analysis of covariance was also used to test differences in nutrient intakes and insulin-resistant phenotypes, including waist circumference, BMI, fasting and $2 \mathrm{~h}$ post-challenge insulin, $\mathrm{ISI}_{0,120}$, HDL-cholesterol, TAG, and systolic and diastolic blood pressure, across the four dietary patterns. Tukey-Kramer's method was used to adjust for the multiple comparisons for all possible pairwise comparisons among the four patterns. Many of the markers of the insulin-resistant phenotypes were positively skewed. Consequently, we performed analysis with and without natural logarithmic data transformation for these markers. Since the finding and statistical inference were the same using both the transformed and untransformed data, we present only the results based on the untransformed data to simplified the data presentation. The covariates included age, sex, waist circumference, smoking, physical activity, treatment of hypertension and total energy intake. Additional adjustment for education (used to capture socioeconomic status ${ }^{(30)}$ ) did not change the results.

In order to examine whether the relationships between dietary patterns and insulin-resistant phenotypes were modified by sex or obesity status (BMI $\geq 30$ or $<30 \mathrm{~kg} / \mathrm{m}^{2}$ ), we examined first-order interactions for each of these associations. Bonferroni adjustment was used to adjust for the multiple comparisons resulting from the examination of these interactions for multiple outcome variables. No statistically significant interactions were observed for any of these outcome variables.

\section{Results}

The mean age of the study participants was 54 years for both men and women. Men had significantly higher waist circumference (98.5 v. $85.6 \mathrm{~cm} ; P<0.01)$, BMI $\left(27.9\right.$ v. $26.3 \mathrm{~kg} / \mathrm{m}^{2}$; $P<0.01)$, physical activity score $(36.1$ v. 33.7 metabolic equivalent task (MET)-h per d; $P<0.01)$, energy intake $(8407$ v. $7306 \mathrm{~kJ} / \mathrm{d} ; P<0.01)$, alcohol intake $(15.5$ v. $7.2 \mathrm{~g} / \mathrm{d}$; $P<0.01)$ and prevalence of hypertension $(19.8$ v. $16.1 \%$; $P=0.01)$ than women. There was no difference between men and women with respect to the proportion of smokers (19.4\% for men and $19.7 \%$ for women).

Four clusters were characterised based on the food or foods that were distinct contributors to total energy intake in each cluster. We named the four clusters as follows: 'Fruits, Reduced Fat Dairy and Whole Grains' pattern (n 577), 'Refined Grains and Sweets' pattern ( $n$ 1846), 'Beer' pattern ( $n$ 242) and 'Soda' pattern ( $n$ 210). The 'Fruits, Reduced Fat Dairy and Whole Grains' pattern was characterised by a relatively higher energy contribution from reduced-fat dairy, whole grains, fruits, vegetables, fish and seafood, and a lower energy contribution from high-fat dairy, meat, chocolate, and sweet baked foods (Table 2). The 'Refined Grains and Sweets' pattern was characterised by a relatively high percentage of energy from sweet baked foods $(9 \%)$ and also contained relatively greater energy contributions from highfat dairy, refined grains, and candy. Energy contributions presented from beer and soda were greatest in the 'Beer' and the 'Soda' patterns, respectively, and they were the only foods to contribute more than $15 \%$ of energy in any of the four patterns. In addition, the 'Beer' and 'Soda' patterns both had a relatively lower energy contribution from many healthier food choices, such as reduced-fat dairy, fruits, vegetables, fish, whole grains; the 'Soda' pattern also had higher contributions from meat, chocolate and miscellaneous sweets. With respect to diet soda consumption, individuals in the 'Soda' pattern consumed significantly less diet soda compared with those in the other dietary patterns.

The four dietary patterns differed significantly with respect to sex, nutrient intakes, dietary glycaemic index and diet quality (Table 2). Participants in the 'Fruits, Reduced Fat Dairy and Whole Grains' pattern were more likely to be female $(67.2 \%)$, and participants in the 'Beer' pattern were more likely to be male $(87.2 \%)$. Participants in the 'Fruits, Reduced Fat Dairy and Whole Grains' pattern had the lowest total energy intake, lowest energy contribution from total fat and saturated fat, lowest cholesterol intake, highest energy contribution from protein intake, highest intake of dietary fibre, highest intake of $\mathrm{K}, \mathrm{Mg}$ and $\mathrm{Ca}$, and highest overall diet quality. Individuals in the 'Refined Grains and Sweets' pattern had the highest energy contribution from total fat, including saturated fat, monounsaturated fat and polyunsaturated fat, and the highest $\mathrm{Na}$ and cholesterol intake. Individuals in the 'Beer' pattern had the highest alcohol intake, and the lowest energy contribution from carbohydrate and sucrose intakes and glycaemic index. Individuals in the 'Soda' pattern had the highest energy intake, highest energy contribution from carbohydrate and sucrose, and glycaemic index. In addition, this dietary pattern had the lowest energy contribution from protein, the lowest intakes of fibre, $\mathrm{K}, \mathrm{Mg}$ and $\mathrm{Ca}$, and the lowest overall diet quality.

Multivariate-adjusted means of insulin-resistant phenotype markers were compared across the four dietary patterns (Table 3). Compared with individuals in the 'Fruits, Reduced Fat Dairy and Whole Grains' pattern, those in the 'Refined Grains and Sweets' pattern had significant higher waist circumference and BMI after adjusting for multiple comparisons and potential confounders, including age, sex, smoking, physical activity, treatment of hypertension and total energy intake. In comparison with individuals in the 'Fruits, Reduced Fat Dairy and Whole Grains' pattern, fasting insulin 
Table 2. Sample characteristic, nutrient intake, glycaemic index and glycaemic load across the four dietary patterns (Mean values with their standard errors)

\begin{tabular}{|c|c|c|c|c|c|c|c|c|}
\hline & \multicolumn{2}{|c|}{$\begin{array}{l}\text { Fruits, Reduced Fat } \\
\text { Dairy and Whole } \\
\text { Grains ( } n 577)\end{array}$} & \multicolumn{2}{|c|}{$\begin{array}{l}\text { Refined Grains } \\
\text { and Sweets } \\
(n 1846)\end{array}$} & \multicolumn{2}{|c|}{$\begin{array}{c}\text { Beer } \\
(n \text { 242) }\end{array}$} & \multicolumn{2}{|c|}{$\begin{array}{l}\text { Soda } \\
\text { (n 210) }\end{array}$} \\
\hline & Mean & SE & Mean & SE & Mean & SE & Mean & SE \\
\hline \multicolumn{9}{|l|}{ Sample characteristics } \\
\hline Age at examination 5 (years) & $55 \cdot 7$ & 0.4 & 53.8 & 0.2 & 51.5 & 0.6 & 51.5 & 0.7 \\
\hline Sex (\% male) & $32 \cdot 8$ & & 41.5 & & $87 \cdot 2$ & & $53 \cdot 3$ & \\
\hline \multicolumn{9}{|l|}{ Nutrients ${ }^{\star}$} \\
\hline Energy $(\mathrm{kJ} / \mathrm{d})$ & $7236^{a} \dagger$ & 106 & $7982^{b}$ & 59 & $7955^{\mathrm{b}}$ & 165 & $8296^{\mathrm{b}} \ddagger$ & 173 \\
\hline Protein (\% energy) & $17 \cdot 5^{\mathrm{a}} \ddagger$ & 0.1 & $17 \cdot 1^{\mathrm{b}}$ & 0.1 & $15 \cdot 0^{\mathrm{c}}$ & 0.2 & $14.4^{\mathrm{c}} \dagger$ & 0.2 \\
\hline Carbohydrate (\% energy) & $55 \cdot 0^{\mathrm{a}}$ & 0.3 & $50 \cdot 0^{b}$ & 0.2 & $45 \cdot 0^{c} \dagger$ & 0.5 & $56 \cdot 2^{a} \ddagger$ & 0.5 \\
\hline Sucrose (\% energy) & $9 \cdot 1^{\mathrm{a}}$ & 0.2 & $10 \cdot 2^{b}$ & 0.1 & $7 \cdot 1^{\mathrm{c}} \dagger$ & 0.3 & $13 \cdot 6^{d} \ddagger$ & 0.3 \\
\hline Fibre $(g / d)$ & $21 \cdot 7^{\mathrm{a}} \ddagger$ & 0.2 & $17 \cdot 3^{\mathrm{b}}$ & 0.1 & $15 \cdot 6^{\mathrm{c}}$ & 0.4 & $12 \cdot 7^{\mathrm{d}} \dagger$ & 0.4 \\
\hline Alcohol (\% energy) & $4 \cdot 4^{\mathrm{a}^{\mathrm{T}}}$ & 0.2 & $2.9^{\mathrm{b}}$ & 0.1 & $14 \cdot 4^{\mathrm{C}} \ddagger$ & 0.3 & $2 \cdot 5^{\mathrm{b}}+$ & 0.3 \\
\hline Total fat (\% energy) & $23 \cdot 4^{\mathrm{a}}+$ & 0.2 & $29.5^{b} \ddagger$ & 0.1 & $25 \cdot 2^{\mathrm{c}}$ & 0.4 & $26 \cdot 6^{\mathrm{d}}$ & 0.4 \\
\hline Saturated fat (\% energy) & $8 \cdot 6^{a}+$ & $0 \cdot 1$ & $11 \cdot 3^{\mathrm{b}} \ddagger$ & $0 \cdot 1$ & $9.9^{c}$ & 0.2 & $10 \cdot 5^{c}$ & 0.2 \\
\hline Monounsaturated fat (\% energy) & $9 \cdot 2^{a} \dagger$ & 0.1 & $12 \cdot 1^{b} \ddagger$ & 0.1 & $10 \cdot 2^{c}$ & 0.2 & $10 \cdot 9^{\mathrm{d}}$ & 0.2 \\
\hline Polyunsaturated fat (\% energy) & $5 \cdot 6^{\mathrm{a}}$ & $0 \cdot 1$ & $6 \cdot 1^{\mathrm{b}} \neq$ & 0.04 & $5 \cdot 1^{c} \dagger$ & $0 \cdot 1$ & $5 \cdot 2^{\mathrm{C}}$ & 0.1 \\
\hline Cholesterol $(\mathrm{mg} / \mathrm{d})$ & $207^{\mathrm{a}}$ & 3 & $237^{b} \neq$ & 2 & $206^{a}+$ & 5 & $217^{\mathrm{a}}$ & 5 \\
\hline $\mathrm{K}(\mathrm{mg} / \mathrm{d})$ & $3515^{\mathrm{a}} \ddagger$ & 24 & $2972^{b}$ & 14 & $2794^{c}$ & 38 & $2419^{d} \dagger$ & 40 \\
\hline $\mathrm{Mg}(\mathrm{mg} / \mathrm{d})$ & $346^{\mathrm{a}} \ddagger$ & 3 & $292^{b}$ & 2 & $319^{c}$ & 4 & $232^{d} \dagger$ & 5 \\
\hline $\mathrm{Ca}(\mathrm{mg} / \mathrm{d})$ & $911^{a} \ddagger$ & 15 & $824^{b}$ & 8 & $683^{c}$ & 23 & $644^{c} \dagger$ & 25 \\
\hline $\mathrm{Na}(\mathrm{mg} / \mathrm{d})$ & $2100^{a}$ & 18 & $2197^{b} \ddagger$ & 10 & $1945^{c}$ & 28 & $1866^{\mathrm{C}} \dagger$ & 29 \\
\hline Glycaemic index & $77 \cdot 8^{\mathrm{a}}$ & 0.2 & $78 \cdot 2^{a}$ & 0.1 & $73.4^{\mathrm{b}} t$ & 0.3 & $81 \cdot 3^{c} \neq$ & 0.3 \\
\hline DGAI & $11 \cdot 1^{a} \ddagger$ & 0.1 & $8 \cdot 6^{\mathrm{b}}$ & 0.1 & $8.0^{\mathrm{c}}$ & 0.1 & $7 \cdot 7^{\mathrm{C}}+$ & 0.2 \\
\hline
\end{tabular}

DGAI, dietary guidelines adherence index.

a,b,c,d Values within a row with unlike superscript letters are significantly different $(P<0.05$; Tukey-Kramer's adjustment for multiple comparisons).

* Adjusted for age, sex and total energy.

† Lowest mean in the row.

$\ddagger$ Highest mean in the row.

concentrations were significantly higher in those individuals in the 'Soda' pattern. In comparison with individuals in the 'Fruits, Reduced Fat Dairy and Whole Grains' pattern, HDL-cholesterol concentrations were significantly higher among those in the 'Beer' pattern. No significant associations were found between dietary pattern and $\mathrm{ISI}_{0,120}, \mathrm{TAG}$, or systolic and diastolic blood pressure.

\section{Discussion}

In this cross-sectional study of healthy adults in the Framingham Offspring Study, four dietary patterns were derived using cluster analysis ('Fruits, Reduced Fat Dairy and Whole Grains', 'Refined Grains and Sweets', 'Beer' and 'Soda'). The 'Refined Grains and Sweets' dietary pattern comprised

Table 3. Insulin-resistant phenotypes across the four dietary patterns

(Mean values with their standard errors)

\begin{tabular}{|c|c|c|c|c|c|c|c|c|}
\hline & \multicolumn{2}{|c|}{$\begin{array}{l}\text { Fruits, Reduced- } \\
\text { Fat Dairy and } \\
\text { Whole Grains } \\
(n 577)\end{array}$} & \multicolumn{2}{|c|}{$\begin{array}{l}\text { Refined Grains } \\
\text { and Sweets } \\
(n 1846)\end{array}$} & \multicolumn{2}{|c|}{$\begin{array}{c}\text { Beer } \\
(n \text { 242) }\end{array}$} & \multicolumn{2}{|c|}{$\begin{array}{l}\text { Soda } \\
(n 210)\end{array}$} \\
\hline & Mean & SE & Mean & SE & Mean & $\mathrm{SE}$ & Mean & SE \\
\hline Waist circumference $(\mathrm{cm})^{\star}$ & $90 \cdot 7^{\mathrm{a}}+$ & 0.5 & $92 \cdot 4^{\mathrm{b}}$ & 0.3 & $91 \cdot 1^{\mathrm{a}, \mathrm{b}}$ & 0.8 & $92 \cdot 5^{\mathrm{a}, \mathrm{b}} \ddagger$ & 0.9 \\
\hline BMI $\left(\mathrm{kg} / \mathrm{m}^{2}\right)^{*}$ & $26 \cdot 6^{\mathrm{a}}$ & 0.2 & $27 \cdot 3^{\mathrm{b}}$ & 0.1 & $26 \cdot 5^{\mathrm{a}, \mathrm{b}} \dagger$ & 0.3 & $27 \cdot 3^{\mathrm{a}, \mathrm{b}} \ddagger$ & 0.3 \\
\hline Fasting insulin $(\mu \mathrm{U} / \mathrm{ml}) \S$ & $28 \cdot 0^{\mathrm{a}}$ & 0.4 & $28 \cdot 8^{a}$ & 0.2 & $27 \cdot 8^{\mathrm{a}} \dagger$ & 0.6 & $31 \cdot 3^{b} \ddagger$ & 0.7 \\
\hline $2 \mathrm{~h}$ post-challenge insulin $(\mu \mathrm{U} / \mathrm{ml}) \S$ & $87 \cdot 3^{a, b}$ & $2 \cdot 7$ & $92 \cdot 8^{a, b}$ & 1.5 & $82 \cdot 4^{a}+$ & $4 \cdot 1$ & $100 \cdot 4^{b} \ddagger$ & $4 \cdot 3$ \\
\hline Insulin sensitivity index $\left(\mathrm{ISI}_{0,120}\right) \S$ & $26 \cdot 8$ & 0.3 & $26 \cdot 6$ & $0 \cdot 2$ & $27 \cdot 6$ & 0.4 & $26 \cdot 5$ & 0.5 \\
\hline HDL-cholesterol $(\mathrm{mmol} / \mathrm{l}) \S$ & $1 \cdot 31^{\mathrm{a}}$ & 0.01 & $1 \cdot 31^{\mathrm{a}}$ & 0.01 & $1.46^{\mathrm{b}} \ddagger$ & 0.02 & $1.25^{\mathrm{a}} \dagger$ & 0.02 \\
\hline TAG $(\mathrm{mmol} / \mathrm{l}) \S$ & 1.53 & 0.04 & 1.54 & 0.02 & 1.55 & 0.07 & 1.59 & 0.07 \\
\hline Systolic blood pressure $(\mathrm{mmHg}) \S \|$ & $123 \cdot 7$ & 0.7 & $121 \cdot 8$ & 0.4 & $124 \cdot 1$ & $1 \cdot 1$ & $123 \cdot 3$ & 1.1 \\
\hline Diastolic blood pressure $(\mathrm{mmHg}) \|$ & $74 \cdot 6$ & $0 \cdot 4$ & $73 \cdot 8$ & 0.2 & $73 \cdot 3$ & 0.7 & 74 & 0.7 \\
\hline
\end{tabular}

a,b,c,d Values within a row with unlike superscript letters are significantly different ( $P<0.05$; Tukey-Kramer's adjustment for multiple comparisons).

* Adjusted for age, sex, smoking, physical activity, treatment of hypertension and total energy.

† Lowest mean in the row.

$\ddagger$ Highest mean in the row.

§Adjusted for age, sex, waist circumference, smoking, physical activity, treatment of hypertension and total energy intake.

$\|$ Excluded those taking blood pressure medications. 
the largest number of individuals, while the 'Soda' pattern comprised the smallest number. The four patterns derived in the present study are similar to those identified using cluster analysis in other American populations ${ }^{(31-34)}$. For example, a 'heart healthy' pattern and an 'empty calorie' pattern, which resemble our 'Fruits, Reduced Fat Dairy and Whole Grains' and 'Soda' patterns, were identified as two of six patterns in women participating at the third examination cycle of the Framingham Offspring Study ${ }^{(33)}$. Consistent with the present study, a 'healthy' and 'alcohol' pattern were identified among five patterns using cluster analysis in 459 men and women in the Baltimore Longitudinal Study of Aging ${ }^{(32)}$. Often, an 'alcohol' pattern is captured in dietary pattern analysis, particularly when men are included in the anal$y \operatorname{sis}^{(32,35,36)}$. In the present study, beer was the predominant contributor to alcohol intake and thus the rationale for naming it the 'Beer' dietary pattern. Although dietary patterns derived in different studies are assigned different names, they often reflect similar food behaviours and nutrient profiles. For instance, our 'Fruits, Reduced Fat Dairy and Whole Grains' pattern is comparable in nutrient content with the 'dark bread, rice and pasta, vegetables' pattern identified in the Insulin Resistance Atherosclerosis Study ${ }^{(34)}$ and a 'milk, cereals, and fruits' pattern identified among elderly Boston area residents ${ }^{(31)}$.

In the present study, compared with individuals in the 'Fruits, Reduced Fat Dairy and Whole Grains' dietary pattern, those in the 'Refined Grains and Sweets' dietary pattern had significantly higher waist circumference and BMI. The 'Refined Grains and Sweets' pattern also had a relatively higher energy contribution from high-fat dairy, meat, refined grains, sweet baked foods, candy, and had a lower energy contribution from reduced-fat dairy, fruits, vegetables and whole grains compared with the 'Fruits, Reduced Fat Dairy and Whole Grains' pattern. This result is consistent with previous findings that a diet high in refined grains, high-fat dairy, meat and sweets, and low in whole grains, fruits and vegetables, was associated with a high risk of obesity ${ }^{(37,38)}$. Prospective studies also found that consuming a diet high in fruits, vegetables, reduced-fat dairy and whole grains was associated with lower increases in BMI and waist circumference ${ }^{(32,39-42)}$. In the present study, none of the dietary patterns was associated with improved blood pressure after the exclusion of those on hypertensive medication.

We found individuals in the 'Soda' pattern had significantly higher fasting insulin concentrations than those in the 'Fruits, Reduced Fat Dairy and Whole Grains' pattern, consistent with our previous finding that the consumption of sugar-sweetened drinks is positively associated with fasting insulin ${ }^{(43)}$. Individuals in the 'Soda' pattern also had a lower dietary fibre intake and a higher dietary glycaemic index. The protective effect of dietary fibre on insulin resistance ${ }^{(4,6,44)}$, the metabolic syndrome $^{(5)}$ and type 2 diabetes mellitus ${ }^{(34,45)}$ has been well documented. McKeown et al. ${ }^{(46)}$ observed that a higher dietary glycaemic index was unfavourably associated with surrogate measures of insulin resistance. In contrast, no association was found between dietary glycaemic index and measures of insulin resistance in either the Insulin Resistance Atherosclerosis Study $^{(6)}$ or the Zutphen Elderly Study ${ }^{(47)}$. Inconsistencies between studies may in part be attributed to the different methods used to determine insulin sensitivity, the different populations studied, and methods of dietary assessment.
Some, but not all, observational studies have found a significant association between sugar-sweetened soft drinks and obesity ${ }^{(48-50)}$. Using cluster analysis, Wirfalt \& Jeffery ${ }^{(28)}$ observed that individuals in the 'soft drinks' pattern had a significantly higher mean BMI compared with individuals in the 'skim milk' and 'meat-cheese' patterns. The 'Soda' pattern had the highest energy contribution from carbohydrate and the highest glycaemic index. Previous studies have demonstrated that high-carbohydrate diets contribute to decreasing HDLcholesterol and increasing TAG concentrations ${ }^{(51,52)}$. Findings from several observational studies have consistently found that glycaemic index is inversely related to HDL-cholesterol concentration $^{(53,54)}$.

Using data from an earlier examination in the Framingham Offspring Cohort, Sonnenberg et al. observed that obese and non-obese women in the 'empty calories' cluster had a higher prevalence of the metabolic syndrome ${ }^{(14)}$. Despite differences in the type of FFQ administered and the defining of the clusters, this dietary pattern characterised by a higher intake of sugar-sweetened beverages and lower intakes of dietary fibre and vegetables is similar to the 'Soda' dietary pattern derived in the present study. Individuals in the 'Soda' pattern displayed several metabolic syndrome abnormalities, i.e. higher waist circumference and TAG and lower HDLcholesterol. However, these differences were not statistically significant compared with the 'Fruits, Reduced Fat Dairy and Whole Grains' pattern, in part due to the small number of subjects in this pattern ( $n$ 210).

Two prospective studies have observed that diet soda consumption is positively associated with the metabolic syndrome ${ }^{(16,50)}$. It is possible that diet soda consumption may be associated with a particular dietary pattern, or alternatively a reflection of a dietary change due to some underlying metabolic disease. In the present study, only those individuals in the 'Soda' cluster differed with respect to diet soda consumption, i.e. they consumed significantly less diet soda compared with the other clusters. We previously found that diet soda consumption was not associated with any surrogate measures of insulin resistance after adjustment for potential confounders $^{(43)}$.

In the present study, individuals in the 'Beer' pattern had significant higher HDL-cholesterol concentration compared with those in the 'Fruits, Reduced Fat Dairy and Whole Grains' pattern. The inverse association between alcohol consumption and CHD has been observed in many observational studies around the world ${ }^{(55)}$. It is estimated that approximately $50 \%$ of the benefit of moderate alcohol consumption could be explained by the direct effect of alcohol on HDLcholesterol $^{(56,57)}$. A meta-analysis of experimental studies found that a dose of $30 \mathrm{~g}$ alcohol per $\mathrm{d}$ increased the concentration of HDL-cholesterol by 39.9 (95\% CI 32.5, 47.3) $\mathrm{mg} / \mathrm{l}^{(58)}$. Although a higher beer intake was associated with a better HDL-cholesterol level, individuals in the 'Beer' pattern had a poorer overall diet, for instance, fewer fruits, vegetables, whole grains, and less reduced-fat dairy. In addition, individuals in the 'Beer' pattern had a significantly lower overall diet quality score compared with individuals in the 'Fruits, Reduced Fat Dairy and Whole Grains' pattern.

Strengths of the present study include its large sample size and inclusion of multiple insulin-resistant phenotypes measured in a clinical setting. The main limitation is the 
cross-sectional study design. Since the diet and insulin-resistant phenotypes data were collected at the same time, the causal relationship cannot be assessed due to uncertainty regarding the timing of exposure and outcome, so further prospective studies are needed to examine the effect of diet on insulin resistance using dietary patterns to characterise exposures. Although we adjusted for physical activity, residual confounding caused by lifestyle behaviours and social economic status factors may have arisen in the present study. The characterisation of the food groups used in the present study is limited by the dietary data obtained by self-reported FFQ. Although the FFQ has limitations in estimating absolute intake for individuals, it is a feasible and valid method to rank (or differentiate) individuals according to their usual diet. Differences in food and nutrient intake profile across clusters, as depicted in Tables 1 and 2, demonstrate the utility of the cluster analysis for discriminating dietary exposure within the cohort. In addition, dietary patterns derived by cluster analysis using FFQ data have been validated against $3 \mathrm{~d}$ food records specifically in the Framingham Offspring Study ${ }^{(33,59)}$. Because the participants of the Framingham Offspring Study are predominantly white Americans, the results from the present study may not be readily generalised to other populations who have different dietary behaviours.

In conclusion, our findings suggest that the consumption of a diet rich in fruits, vegetables, whole grains and reduced-fat dairy protects against insulin-resistant phenotypes; displacing these healthy choices with meat, refined grains, high-fat dairy, sweet baked foods, candy and sugar-sweetened soda promotes insulin-resistant phenotypes.

\section{Acknowledgements}

The present study was supported in part by the US Department of Agriculture, under agreement no. 58-1950-7-707, and with support from the Framingham Heart Study of the National Heart Lung and Blood Institute of the National Institutes of Health (contract no. N01-HC-25195). J. B. M. is supported by the National Institute of Diabetes and Digestive and Kidney Diseases (NIDDK) K24 DK080140. R. S. V. is supported in part by $2 \mathrm{~K} 24 \mathrm{HL} 04334$. N. M. is supported in part by a grant from the General Mills Bell Institute of Health and Nutrition.

Any opinions, findings, conclusions, or recommendations expressed in this publication are those of the author(s) and do not necessarily reflect the view of the US Department of Agriculture.

We are grateful to the Framingham Study participants and staff for data collection. We thank Gail Rogers for data management.

The authors' responsibilities were as follows. E. L. designed and performed the statistical analyses and drafted the manuscript. P. F. J., N. M. M. and P. K. N. contributed to the design of the analyses, the interpretation of the data, and the writing of the manuscript. J. B. M., P. A. Q. and R. S. V. contributed to the interpretation of the data and the writing of the manuscript. R. B. D. served as liaison with Framingham Study investigators, provided data, and contributed to the interpretation of the data and the writing of the manuscript. All authors participated in the revision and approval of the manuscript.
N. M. M. has served as an independent consultant for Cadbury. None of the other authors has any conflict of interest.

The supplementary material for this article can be found at http://www.journals.cambridge.org/bjn

\section{References}

1. Mlinar B, Marc J, Janez A, et al. (2007) Molecular mechanisms of insulin resistance and associated diseases. Clin Chim Acta 375, 20-35.

2. Rader DJ (2007) Effect of insulin resistance, dyslipidemia, and intra-abdominal adiposity on the development of cardiovascular disease and diabetes mellitus. Am J Med 120, S12-S18.

3. Hu FB, van Dam RM \& Liu S (2001) Diet and risk of type II diabetes: the role of types of fat and carbohydrate. Diabetologia 44, 805-817.

4. McKeown NM, Meigs JB, Liu S, et al. (2002) Whole-grain intake is favorably associated with metabolic risk factors for type 2 diabetes and cardiovascular disease in the Framingham Offspring Study. Am J Clin Nutr 76, 390-398.

5. McKeown NM, Meigs JB, Liu S, et al. (2004) Carbohydrate nutrition, insulin resistance, and the prevalence of the metabolic syndrome in the Framingham Offspring Cohort. Diabetes Care 27, 538-546.

6. Liese AD, Schulz M, Fang F, et al. (2005) Dietary glycemic index and glycemic load, carbohydrate and fiber intake, and measures of insulin sensitivity, secretion, and adiposity in the Insulin Resistance Atherosclerosis Study. Diabetes Care 28, $2832-2838$.

7. Rumawas ME, McKeown NM, Rogers G, et al. (2006) Magnesium intake is related to improved insulin homeostasis in the Framingham Offspring Cohort. J Am Coll Nutr 25, 486-492.

8. Jacques PF \& Tucker KL (2001) Are dietary patterns useful for understanding the role of diet in chronic disease? Am J Clin Nutr 73, 1-2.

9. Hu FB (2002) Dietary pattern analysis: a new direction in nutritional epidemiology. Curr Opin Lipidol 13, 3-9.

10. Hu FB, Rimm E, Smith-Warner SA, et al. (1999) Reproducibility and validity of dietary patterns assessed with a foodfrequency questionnaire. Am J Clin Nutr 69, 243-249.

11. Esmaillzadeh A, Kimiagar M, Mehrabi Y, et al. (2007) Dietary patterns, insulin resistance, and prevalence of the metabolic syndrome in women. Am J Clin Nutr 85, 910-918.

12. Panagiotakos DB, Pitsavos C, Skoumas Y, et al. (2007) The association between food patterns and the metabolic syndrome using principal components analysis: the ATTICA Study. J Am Diet Assoc 107, 979-987.

13. Williams DE, Prevost AT, Whichelow MJ, et al. (2000) A crosssectional study of dietary patterns with glucose intolerance and other features of the metabolic syndrome. Br J Nutr 83, 257-266.

14. Sonnenberg L, Pencina M, Kimokoti R, et al. (2005) Dietary patterns and the metabolic syndrome in obese and non-obese Framingham women. Obes Res 13, 153-162.

15. Wirfalt E, Hedblad B, Gullberg B, et al. (2001) Food patterns and components of the metabolic syndrome in men and women: a cross-sectional study within the Malmo Diet and Cancer cohort. Am J Epidemiol 154, 1150-1159.

16. Lutsey PL, Steffen LM \& Stevens J (2008) Dietary intake and the development of the metabolic syndrome: the Atherosclerosis Risk in Communities study. Circulation 117, 754-761.

17. Nettleton JA, Steffen LM, Mayer-Davis EJ, et al. (2006) Dietary patterns are associated with biochemical markers of inflammation and endothelial activation in the Multi-Ethnic Study of Atherosclerosis (MESA). Am J Clin Nutr 83, 1369-1379. 
18. Feinleib M, Kannel WB, Garrison RJ, et al. (1975) The Framingham Offspring Study. Design and preliminary data. Prev Med 4, $518-525$.

19. Rimm EB, Giovannucci EL, Stampfer MJ, et al. (1992) Reproducibility and validity of an expanded self-administered semiquantitative food frequency questionnaire among male health professionals. Am J Epidemiol 135, 1114-1136.

20. Willett WC, Sampson L, Stampfer MJ, et al. (1985) Reproducibility and validity of a semiquantitative food frequency questionnaire. Am J Epidemiol 122, 51-65.

21. Fogli-Cawley JJ, Dwyer JT, Saltzman E, et al. (2006) The 2005 Dietary Guidelines for Americans Adherence Index: development and application. J Nutr 136, 2908-2915.

22. World Health Organization (1980) WHO Expert Committee on Diabetes Mellitus: Second Report. Geneva: World Health Organization.

23. Gutt M, Davis CL, Spitzer SB, et al. (2000) Validation of the insulin sensitivity index $\left(\operatorname{ISI}_{(0,120)}\right)$ : comparison with other measures. Diabetes Res Clin Pract 47, 177-184.

24. McNamara JR \& Schaefer EJ (1987) Automated enzymatic standardized lipid analyses for plasma and lipoprotein fractions. Clin Chim Acta 166, 1-8.

25. Warnick GR, Benderson J \& Albers JJ (1982) Dextran sulfate$\mathrm{Mg}^{2+}$ precipitation procedure for quantitation of high-densitylipoprotein cholesterol. Clin Chem 28, 1379-1388.

26. Kannel WB \& Sorlie P (1979) Some health benefits of physical activity. The Framingham Study. Arch Intern Med 139, 857-861.

27. Newby PK, Muller D \& Tucker KL (2004) Associations of empirically derived eating patterns with plasma lipid biomarkers: a comparison of factor and cluster analysis methods. Am J Clin Nutr 80, 759-767.

28. Wirfalt AK \& Jeffery RW (1997) Using cluster analysis to examine dietary patterns: nutrient intakes, gender, and weight status differ across food pattern clusters. J Am Diet Assoc 97, $272-279$.

29. Willett WC, Howe GR \& Kushi LH (1997) Adjustment for total energy intake in epidemiologic studies. Am J Clin Nutr $\mathbf{6 5}$, 1220S-1231S.

30. Cagney KA \& Lauderdale DS (2002) Education, wealth, and cognitive function in later life. J Gerontol B Psychol Sci Soc Sci 57, P163-P172.

31. Tucker KL, Dallal GE \& Rush D (1992) Dietary patterns of elderly Boston-area residents defined by cluster analysis. $J$ Am Diet Assoc 92, 1487-1491.

32. Newby PK, Muller D, Hallfrisch J, et al. (2003) Dietary patterns and changes in body mass index and waist circumference in adults. Am J Clin Nutr 77, 1417-1425.

33. Millen BE, Quatromoni PA, Copenhafer DL, et al. (2001) Validation of a dietary pattern approach for evaluating nutritional risk: the Framingham Nutrition Studies. J Am Diet Assoc 101, 187-194.

34. Liese AD, Schulz M, Moore CG, et al. (2004) Dietary patterns, insulin sensitivity and adiposity in the Multi-Ethnic Insulin Resistance Atherosclerosis Study population. Br J Nutr 92, 973-984.

35. Tucker KL, Chen H, Hannan MT, et al. (2002) Bone mineral density and dietary patterns in older adults: the Framingham Osteoporosis Study. Am J Clin Nutr 76, 245-252.

36. Millen BE, Quatromoni PA, Gagnon DR, et al. (1996) Dietary patterns of men and women suggest targets for health promotion: the Framingham Nutrition Studies. Am J Health Promot 11, 42-53.

37. Esmaillzadeh A \& Azadbakht L (2008) Major dietary patterns in relation to general obesity and central adiposity among Iranian women. J Nutr 138, 358-363.

38. Murtaugh MA, Herrick JS, Sweeney C, et al. (2007) Diet composition and risk of overweight and obesity in women living in the southwestern United States. J Am Diet Assoc 107, $1311-1321$.
39. Quatromoni PA, Copenhafer DL, D’Agostino RB, et al. (2002) Dietary patterns predict the development of overweight in women: The Framingham Nutrition Studies. J Am Diet Assoc 102, 1239-1246.

40. Newby PK, Muller D, Hallfrisch J, et al. (2004) Food patterns measured by factor analysis and anthropometric changes in adults. Am J Clin Nutr 80, 504-513.

41. Schulze MB, Fung TT, Manson JE, et al. (2006) Dietary patterns and changes in body weight in women. Obesity 14, 1444-1453.

42. Mendez MA, Popkin BM, Jakszyn P, et al. (2006) Adherence to a Mediterranean diet is associated with reduced 3-year incidence of obesity. J Nutr 136, 2934-2938.

43. Yoshida M, McKeown NM, Rogers G, et al. (2007) Surrogate markers of insulin resistance are associated with consumption of sugar-sweetened drinks and fruit juice in middle and olderaged adults. J Nutr 137, 2121-2127.

44. Buyken AE \& Liese AD (2005) Dietary glycemic index, glycemic load, fiber, simple sugars, and insulin resistance: the Inter99 Study: response to Lau et al. Diabetes Care 28, 2986-2987.

45. Salmeron J, Manson JE, Stampfer MJ, et al. (1997) Dietary fiber, glycemic load, and risk of non-insulin-dependent diabetes mellitus in women. JAMA 277, 472-477.

46. McKeown NM, Meigs JB, Liu S, et al. (2009) Dietary carbohydrates and cardiovascular disease risk factors in the Framingham Offspring Cohort. J Am Coll Nutr (In the Press).

47. van Dam RM, Visscher AW, Feskens EJ, et al. (2000) Dietary glycemic index in relation to metabolic risk factors and incidence of coronary heart disease: the Zutphen Elderly Study. Eur J Clin Nutr 54, 726-731.

48. Ludwig DS, Peterson KE \& Gortmaker SL (2001) Relation between consumption of sugar-sweetened drinks and childhood obesity: a prospective, observational analysis. Lancet 357, 505-508.

49. Schulze MB, Manson JE, Ludwig DS, et al. (2004) Sugar-sweetened beverages, weight gain, and incidence of type 2 diabetes in young and middle-aged women. JAMA 292, 927-934.

50. Dhingra R, Sullivan L, Jacques PF, et al. (2007) Soft drink consumption and risk of developing cardiometabolic risk factors and the metabolic syndrome in middle-aged adults in the community. Circulation 116, 480-488.

51. Reaven GM (1997) Do high carbohydrate diets prevent the development or attenuate the manifestations (or both) of syndrome X? A viewpoint strongly against. Curr Opin Lipidol 8, 23-27.

52. Reaven GM (2005) The insulin resistance syndrome: definition and dietary approaches to treatment. Annu Rev Nutr 25, 391-406.

53. Frost G, Leeds AA, Dore CJ, et al. (1999) Glycaemic index as a determinant of serum HDL-cholesterol concentration. Lancet 353, 1045-1048.

54. Ford ES \& Liu S (2001) Glycemic index and serum high-density lipoprotein cholesterol concentration among us adults. Arch Intern Med 161, 572-576.

55. Lucas DL, Brown RA, Wassef M, et al. (2005) Alcohol and the cardiovascular system research challenges and opportunities. J Am Coll Cardiol 45, 1916-1924.

56. Langer RD, Criqui MH \& Reed DM (1992) Lipoproteins and blood pressure as biological pathways for effect of moderate alcohol consumption on coronary heart disease. Circulation 85, 910-915.

57. Rimm EB \& Moats C (2007) Alcohol and coronary heart disease: drinking patterns and mediators of effect. Ann Epidemiol 17, S3-S7.

58. Rimm EB, Williams P, Fosher K, et al. (1999) Moderate alcohol intake and lower risk of coronary heart disease: meta-analysis of effects on lipids and haemostatic factors. BMJ 319, 1523-1528.

59. Quatromoni PA, Copenhafer DL, Demissie S, et al. (2002) The internal validity of a dietary pattern analysis. The Framingham Nutrition Studies. J Epidemiol Comm Health 56, 381-388. 